\title{
Faas, Thorsten, Oscar W. Gabriel, und Jürgen Maier (Hrsg.) (2020): Politikwissenschaftliche Einstellungs- und Verhaltensforschung. Handbuch für Wissenschaft und Studium
}

\author{
Nomos: Baden-Baden. 718 Seiten. $98 €$
}

\section{Martin Elff $\mathbb{D}$}

Angenommen: 21. Dezember 2020 / Online publiziert: 12. Januar 2021

(C) Der/die Autor(en) 2021

Auf nicht weniger als 718 Seiten und in nicht weniger als 30 Kapiteln befasst sich das Handbuch „Politikwissenschaftliche Einstellungs- und Verhaltensforschung“ mit vielen der wichtigsten Themen dieses Teilbereichs der Politikwissenschaft sowie einigen ausgewählten Methoden der Datenerhebung. Die 30 Kapitel des Bandes sind, abgesehen von einem Einleitungskapitel, das die Entstehungsgeschichte der Subdisziplin beschreibt, in fünf Teile gliedert, die jeweils fünf bis sieben Kapitel umfassen. Diese Buchteile befassen sich mit politischer Kommunikation, politischen Einstellungen, politischer Partizipation, Wahlverhalten und Methoden. Die einzelnen Kapitel folgen mit geringfügigen Abweichungen einem einheitlichen Aufbauschema: Ein erster Abschnitt beschreibt die Entstehung des jeweiligen Forschungsfeldes, ein zweiter Abschnitt führt in die grundlegenden Begriffe ein, ein dritter Abschnitt beschreibt wichtige empirische Befunde, ein vierter Abschnitt diskutiert Erklärungsansätze für diese Befunde und ein abschließender fünfter Abschnitt zeigt die noch offenen Fragen auf und weist auf Kontroversen hin.

Ziel des Handbuchs ist laut den Herausgebern, ,dem Fachpublikum und einer breiteren, interessierten Leserschaft einen umfassenden Überblick über den aktuellen Stand der internationalen politikwissenschaftlichen Einstellungs- und Verhaltensforschung zu geben“ (S. 72). Dies ist allerdings ein Ziel, das sich kaum zur Gänze erreichen lässt. Entsprechend gestehen die Herausgeber ein, dass der Band ,unvermeidliche Lücken im Überblick über die Felder“ aufweist, die zwar auch „Auswahlentscheidungen, die die subjektiven Präferenzen der Entscheidenden widerspiegeln“, aber auch auf außerhalb des Einflusses der Herausgeber liegende Faktoren zurückgehen (S. 73). Allerdings kann weniger von Auslassungen in der Behandlung der Themenfelder gesprochen werden als vielmehr von Schlagseiten, die - so viel muss

M. Elff (ه)

Zeppelin Universität, Friedrichshafen, Deutschland

E-Mail: martin.elff@zu.de 
zugestanden werden - wohl kaum vermeidbar sind. Es ist kaum möglich, in auch nur einem Themenfeld den Forschungsstand sowohl erschöpfend als auch in der gebotenen Kürze und Verständlichkeit darzustellen. Zwar schließt die Diskussion der grundlegenden Konzepte in der überwiegenden Mehrzahl der Kapitel an die internationale Literatur an, jedoch kann sie zumeist nur einen Überblick über den empirischen Forschungsstand mit Bezug auf Deutschland vermitteln.

In einigen Kapiteln rückt aber der Überblick über das jeweilige Teilgebiet gegenüber der Darstellung der aktuellen Forschungsinteressen und -befunde der Autorinnen und Autoren in den Hintergrund. Zum Beispiel nimmt im Kapitel „Politischer Protest und Konsum“ die Diskussion von politischem Konsum mindestens ebenso viel Raum ein wie politischer Protest, obgleich die Erforschung von politischem Protest eine lange Tradition hat, während politischer Konsum ein eher randständiges Thema ist. Wenn es denn schon behandelt wird, sollte zumindest auch diskutiert werden, ob ethisch motiviertes Kaufverhalten überhaupt als politische Aktivität gelten kann. Andere Kapitel enthalten Originalauswertungen der Autorinnen oder Autoren, die eher der Präsentation eigener Forschung als der Illustration des Themenfelds dienen. Eine stärker kritisch-reflektierende Perspektive, aus der zum Beispiel in Arzheimers Kapitel zum politischen Extremismus, Krohs Kapitel zu Parteiidentifikation oder Ohrs Kapitel zu Kandidatenorientierungen die im jeweiligen Forschungsfeld gängigen Annahmen und verbreiteten Thesen betrachtet werden, hätte mehreren anderen Kapiteln nicht geschadet.

Wenig zufriedenstellend ist die Auswahl der im Handbuch behandelten Methoden. Ihre Auswahl und Darstellung ist ziemlich deutlich kommunikationswissenschaftlich geprägt. Eine klaffende Lücke ist das Fehlen eines Kapitels zu Umfragen, die in der politikwissenschaftlichen Einstellungs- und Verhaltensforschung nach wie vor eine zentrale Rolle spielen. Gerne hätte man mehr darüber gelesen, ob und wie sich z.B. das Problem schwindender Rücklaufquoten lösen oder bewältigen lässt oder welche Potenziale das Internet für politikwissenschaftliche Umfragen hat. Weiterhin hätte ein Kapitel zur Kausalinferenz - ein Thema, das in der internationalen Politikwissenschaft viel Aufmerksamkeit erhält - dem Handbuch sicherlich nicht geschadet. Ein Kapitel zu Experimenten ist zwar vorhanden, allerdings ist die dort behandelte internationale Literatur überwiegend mindestens zehn Jahre alt, obwohl die experimentelle Politikwissenschaft vor allem in jüngeren Jahren einen Boom erlebt. Zum Beispiel gibt es das Journal of Experimental Political Science erst seit 2014.

Trotz der erwähnten Abstriche ist die Lektüre des Handbuchs durchaus ein Gewinn. Während einige Kapitel (in der Regel notgedrungen) sehr dicht geschrieben sind, gelingt es fast allen Kapiteln, ihre jeweiligen Themenbereiche verständlich zu machen. Für Wissenschaftlerinnen und Wissenschaftler sowie fortgeschrittene Studierende kann das Handbuch sicherlich einen guten Einstieg in für sie neue Themenbereiche bieten. Wer aber einen fundierten Überblick über den internationalen Stand der Forschung gewinnen will, sollte auch einen Blick in englischsprachige Handbücher werfen, wie zum Beispiel das von Arzheimer, Evans und Lewis-Beck (2017) herausgegebene SAGE Handbook of Electoral Behaviour. 
Funding Open Access funding enabled and organized by Projekt DEAL.

Open Access Dieser Artikel wird unter der Creative Commons Namensnennung 4.0 International Lizenz veröffentlicht, welche die Nutzung, Vervielfältigung, Bearbeitung, Verbreitung und Wiedergabe in jeglichem Medium und Format erlaubt, sofern Sie den/die ursprünglichen Autor(en) und die Quelle ordnungsgemäß nennen, einen Link zur Creative Commons Lizenz beifügen und angeben, ob Änderungen vorgenommen wurden.

Die in diesem Artikel enthaltenen Bilder und sonstiges Drittmaterial unterliegen ebenfalls der genannten Creative Commons Lizenz, sofern sich aus der Abbildungslegende nichts anderes ergibt. Sofern das betreffende Material nicht unter der genannten Creative Commons Lizenz steht und die betreffende Handlung nicht nach gesetzlichen Vorschriften erlaubt ist, ist für die oben aufgeführten Weiterverwendungen des Materials die Einwilligung des jeweiligen Rechteinhabers einzuholen.

Weitere Details zur Lizenz entnehmen Sie bitte der Lizenzinformation auf http://creativecommons.org/ licenses/by/4.0/deed.de. 